\title{
PRESENTATION AND OUTCOME O ACUTE KIDNEY INJURY IN A TERTIARY MILITARY HOSPITAL OF BANGLADESH
}

\author{
MAMUN MOSTAFI, MAJ AZIZUN NESSA, MD AMZAD HOSSAIN FAKIR, MD ABDUL QUDDUS BHUYIAN, \\ AKM MIJANUR RAHMAN
}

\begin{abstract}
Introduction: Acute kidney injury (AKI) is a common condition and its incidence is increasing. No study has been done so far on this subject in the Armed Forces. This retrospective study is therefore, to find out the incidence, etiology, predisposing factors, diagnostic approach, clinical course and finally outcome of the patients with AKI in this country, in a selected group of patients.

Methods: This retrospective cross sectional study was conducted in Combined Military Hospital, Dhaka, from July 2007 to July 2011. Total 105 cases were included in this study. All were adult and their age of distribution was 18-80 years. Cases were studied in terms of etiology, mode of presentation, laboratory findings, management and response to treatment or outcome.

Results: Mean age was 47.24+ 18.35 years. Male, female ratio was 3:2. Hypovolemia was the major (23.8\%) etiological factor of AKI in this study due to acute gastroenteritis, $14.28 \%$ cases were due to different non-steroidal anti inflammatory drugs (NSAIDs), 9.52\% cases due to rhabdomyolysis following physical assault and vigorous exercise, 7.61\% cases were due to septicaemia, $8.57 \%$ cases were due to glomerulonephritis and $6.66 \%$ cases due to acute pyelonephritis. $6.66 \%$ cases developed AKI due to falciparum malaria and $5.71 \%$ due to obstructive uropathy. Other causes of AKI in this study are -contrast induced nephropathy 3.8\%,postoperative AKI 4.76\%,AKI due to vasculitis $3.8 \%, 2.85 \%$ cases due to HELLP syndrome,0.95\% case due to snake bite and $0.95 \%$ case due to thrombotic thrombocytopenic purpura(TTP). Oliguria (66.66\%) and oedema (64.76\%) were the commonest presentation in this study. Mean blood urea and serum creatinine level on admission being $9134 \mathrm{mg} / \mathrm{dl}$ and $4.292 .55 \mathrm{mg} / \mathrm{dl}$ respectively. Haemodialysis was done in 44 cases, continuous renal replacement therapy (CRRT-continuous venovenous haemodialysis) was given in 6 patients, peritoneal dialysis in 5 patients and 49 cases were managed with conservative therapy alone. Complete recovery occurred in 88 patients, 8 had recovery with residual renal impairment, 6 patients developed end stage renal disease (ESRD) and 3 patients died due to septicaemia and multi-organ failure (MOF).
\end{abstract}

Conclusion: Most of the cases of AKI are preventable if we can take due care of some common health problems like gastroenteritis and at the same time outcome can be rewarding if we can ensure early reporting, quick diagnosis and appropriate management

\section{Introduction}

Acute kidney Injury (AKI) is one of the most dreadful disease and therefore, a challenging problem in our country. AKI has now replaced the term acute renal failure and a universal definition and staging system has been proposed to allow earlier detection and management of AKI. ${ }^{1}$ The new terminology enables healthcare professionals to consider the disease as a spectrum of injury. This spectrum extends from less severe forms of injury to more advanced injury when acute kidney failure may require renal replacement therapy (RRT). ${ }^{1}$ Clinically AKI is characterized by a rapid reduction in kidney function resulting in a failure to maintain fluid, electrolyte and acid-base homoeostasis. ${ }^{2}$ Previously there have been many different definitions of AKI used in the literature which has made it difficult to determine the epidemiology and outcomes of AKI. Over recent years there has been increasing recognition that relatively small rises in serum creatinine level in a variety of clinical settings may be associated with worse outcome. ${ }^{3,}{ }^{4}$ Over the years, the etiology,presentation and management of AKI has been changed .The study was designed to observe the recent trends of AKI and its outcome in a tertiary military hospital.

\section{Materials and Methods}

This retrospective cross sectional study was conducted in Combined Military Hospital, Dhaka,

Department of Nephrology, Armed Force Medical College, Dhaka

Bangladesh J Medicine 2011; 22 : 35-40 
Bangladesh from July 2007 to July 2011. Total 105 cases were included in this study coming from specific group of population that is Bangladesh Armed Forces. Cases were studied in terms of etiology, mode of presentation, laboratory findings, management and response to treatment or outcome. Patients already known to have CKD, with sudden deterioration of renal function due to some insult or patient with small sized kidneys as seen by ultrasonogram were excluded from this study.

\section{Results and Findings}

Most patients (33.33\%) were from 31-45 years age group and male female ratio was 3:2 (table-I). Hypovolemia was the most common (23.8\%) etiological factor of AKI which was mainly due to acute gastroenteritis. Other important causes of AKI were NSAIDs (14.28\%), rhabdomyolysis (9.52\%), septicaemia (7.61\%), glomerulonephritis $(8.57 \%)$, acute pyelonephrirtis $(6.66 \%)$, falciparum malaria $(6.66 \%)$ etc (table-II ). Oliguria was the most common $(66.66 \%)$ mode of presentation whereas $22.85 \%$ were nonoliguric and $6.66 \%$ were anuric . Diarrhoea were presenting symptoms in $23.8 \%$ patients and $32.38 \%$ had shortness of breathing. $66.66 \%$ had oedema and $.085 \%$ had acidotic breath . $17.14 \%$ were hypertensive and $16.19 \%$ were in shock(table-III).Laboratory findings showed protienuria was present in $41.90 \%$ cases, $40 \%$ had WBC, $32.88 \%$ had RBC and $26.66 \%$ had RBC cast in their urine(table-IV). Mean blood urea was $91 \pm 34 \mathrm{mg} / \mathrm{dl}$ whereas serum creatinine was $4.29 \pm 2.55 \mathrm{mg} / \mathrm{dl}$ at presentation (table V), which gradually decreased with time. Mean duration of hospital stay was $19 \pm 10.2$ days. $42.85 \%$ received haemodialysis, $5.71 \%$ received CRRT whereas peritoneal dialysis was given in $4.76 \%$ patient. 49 patients $(46.66 \%)$ received conservative management only(table VII). With these management 88 patients $(83.80 \%)$ completely recovered. 8 patients survived with some residual renal impairment, 6 developed end stage renal disease (ESRD) three patients expired due to septicaemia or MOF(table II).

Table-I

Age and Sex Distribution of Patients ( $n=105)$.

\begin{tabular}{lccc}
\hline $\begin{array}{l}\text { Age group } \\
\text { (in yrs) }\end{array}$ & Male & Female & $\begin{array}{c}\text { Total } \\
\text { (percentage) }\end{array}$ \\
\hline $18-30$ & 18 & 6 & $24(22.86 \%)$ \\
$31-45$ & 21 & 14 & $35(33.33 \%)$ \\
$46-60$ & 08 & 10 & $18(17.14 \%)$ \\
$>60$ & 16 & 12 & $28(26.67 \%)$ \\
\hline Total & $63(60 \%)$ & $42(40 \%)$ & $100 \%$ \\
\hline
\end{tabular}

Table -II

Etiology and Outcome of AKI $(n=105)$.

\begin{tabular}{|c|c|c|c|c|}
\hline Cause & Number (\%) & Complete recovery & Progression to CKD & Death \\
\hline Hypovolemia & $25(23.8 \%)$ & 25 & - & - \\
\hline NSAIDs & $15(14.28 \%)$ & 11 & 4 & - \\
\hline Rhabdomyolysis & $10(9.52 \%)$ & 10 & - & - \\
\hline Septicaemia & $8(7.61 \%)$ & 6 & 1 & 1 \\
\hline Glomerulonephritis & $9(8.57 \%)$ & 8 & - & - \\
\hline Acute pyelonephritis & $7(6.66 \%)$ & 7 & - & 1 \\
\hline Falciparum Malaria & 7 (6.66\%) & 7 & - & - \\
\hline Obstructive Uropathy & $6(5.71 \%)$ & 5 & 1 & - \\
\hline Contrast induced -Nephropathy & $4(3.80 \%)$ & 2 & 2 & - \\
\hline Post operative & $5(4.76 \%)$ & 3 & 2 & - \\
\hline Vasculitis & $4(3.80 \%)$ & - & 4 & 1 \\
\hline HELLP syndrome & $3(2.85 \%)$ & 3 & - & - \\
\hline Snake Bite & 1 (0.95\%) & 1 & - & - \\
\hline TTP & $1(0.95 \%)$ & 1 & - & - \\
\hline
\end{tabular}


Table-III Mode of Presentation ( $n=105)$.

\begin{tabular}{lcc}
\hline *Presentation & Number of patient & Percentage \\
\hline Oliguria & 70 & $66.66 \%$ \\
Non-oliguric & 24 & $22.85 \%$ \\
Anuria & 25 & $6.66 \%$ \\
Diarrhoea & 34 & $23.8 \%$ \\
Shortness of breath & 70 & $32.38 \%$ \\
Oedema & 5 & $66.66 \%$ \\
Haematuria & 17 & $.047 \%$ \\
Shock & 8 & $16.19 \%$ \\
Hypotension & 18 & $7.61 \%$ \\
Hypertension & 9 & $17.14 \%$ \\
Acidotic breath & 8 & $.085 \%$ \\
Loin Pain & 13 & $7.61 \%$ \\
Drowsiness & 6 & $12.4 \%$ \\
Convulsion & & $.057 \%$ \\
\hline
\end{tabular}

*Findings overlap.

Table-IV

Findings of Routine Urine Analysis(n=105).

\begin{tabular}{lcc}
\hline *Findings & Number of patients & Percentage \\
\hline Normal & 48 & $45.71 \%$ \\
Proteinuria & 44 & $41.90 \%$ \\
WBC in urine & 42 & $40 \%$ \\
RBC in urine & 34 & $32.38 \%$ \\
RBC casts & 28 & $26.66 \%$ \\
Granular casts & 39 & $37.14 \%$ \\
\hline
\end{tabular}

*Findings overlap.

Table-V

Blood Biochemistry(at presentation).

\begin{tabular}{lcc}
\hline Tests & Mean & $\begin{array}{c}\text { Standard } \\
\text { deviation }\end{array}$ \\
\hline Blood Urea $(\mathrm{mg} / \mathrm{dl})$ & 91 & 34 \\
Serum Creatinine $(\mathrm{mg} / \mathrm{dl})$ & 4.29 & 2.55 \\
Serum Sodium $(\mathrm{mmol} / \mathrm{l})$ & 142 & 2.46 \\
Serum Potassium $(\mathrm{mmol} / \mathrm{l})$ & 4.44 & 1.69 \\
Serum chloride $(\mathrm{mmol} / \mathrm{l})$ & 99.5 & 4.09 \\
Serum calcium $(\mathrm{mg} / \mathrm{dl})$ & 8.6 & 1.5 \\
Serum phosphate $(\mathrm{mg} / \mathrm{dl})$ & 5.5 & 1.2 \\
Serum uric acid $(\mathrm{mg} / \mathrm{dl})$ & 7.8 & 1.8 \\
\hline
\end{tabular}

Table -VI

Other investigations.

\begin{tabular}{llc}
\hline Investigations & *Findings & $\begin{array}{c}\text { No of patients } \\
\text { (Percentage) }\end{array}$ \\
\hline CXR & Pulmonary oedema & $30(28.57 \%)$ \\
USG of KUB & Renal/ureteric calculi & $6(5.71) \%$ \\
Plain X-Ray KUB & Renal/ureteric calculi & $6(5.71) \%$ \\
ECG & Features of hyperkalaemia $4(3.81 \%)$ \\
Urine C/S & Growth of organism & $7(6.66 \%)$ \\
HBsAg & Positive & $7(6.66 \%)$ \\
\hline
\end{tabular}

*Findings overlap.

Table-VII

Types of Management ( $n=105)$.

\begin{tabular}{lcc}
\hline Type of therapy & No. of patients & Percentage \\
\hline Haemodialysis & 45 & $42.85 \%$ \\
CRRT(CVVHD) & 06 & $05.71 \%$ \\
Peritoneal dialysis & 05 & $04.76 \%$ \\
Conservative only & 49 & $46.66 \%$ \\
\hline
\end{tabular}

\section{Discussion}

The result of the present study showed information regarding the presentation and prognosis of acute renal failure in Bangladesh Armed Forces. All the clinical parameters, available biochemical estimations, management and prognosis of the patients with acute renal failure have been studied in this series.

The clinical spectrum of acute renal failure in this study is clearly differentiable from those of the published western reports. ${ }^{5,6,7}$ The age of the patients included in this study ranges from 18-80 years but the incidence of AKI found to be high in 31-45 years age group (33.33\%).

In contrast to developed countries where most cases of AKI are related to trauma and multi-organ failure, AKI in developing countries is often related to medical cause. ${ }^{6}$ In this study hypovolemia mostly due to diarrhoea and vomiting was the leading cause (23.8\%) of AKI . The next common etiologies were NSAIDs, contrast AKI and rhabdomyolysis. This is comparable to observations in India ${ }^{7}$ where over $60 \%$ of AKI cases are related to gastroenteritis and also to our country. ${ }^{8,9}$

Trauma due to road traffic and industrial accidents, cardiovascular surgery and cardiogenic shock appeared to proceed acute kidney injury in $60 \%$ cases 
in the developed countries is in sharp contrast to this study. ${ }^{8,10}$

Eighteen (17.14\%) patients in this study developed AKI following the ingestion of NSAIDs which they took for pain of different origin. NSAIDs induced nephropathy and AKI is not uncommon in military personnel .They usually take different NSAIDs for exercise induced injury. However, these patients with AKI in this study fully recovered after withdrawal of drug along with adequate hydration and supportive measures. AKI due to various drugs \& chemicals occurs with increasing frequency in the developed countries. Increased risk involved in both short and long-term therapy and is slightly greater among users of high doses ${ }^{11}$. Drug induced AKI is not uncommon in this country. Superstition, self medication and exploitation of illiterate people by quacks are a potential risk in this country which needs to be prevented. NSAIDs users have a three fold greater risk for developing a first-ever diagnosis of clinical AKI compared with non-NSAIDs users in the general population. NSAIDs should be used with special caution in patients with diabetes mellitus, hypertension and heart failure. ${ }^{12}$

Twelve (11.42\%) patients developed AKI due to rhabdomyolysis following physical assault and vigorous exercise which is not uncommon in military personnel which simulate to other studies. ${ }^{13,14,15}$

Septic AKI is common during the first 24 hours after ICU admission. Patients with septic AKI are generally sicker, with a higher burden of illness and have greater abnormalities in acute physiology compared with patients with nonseptic AKI. ${ }^{16}$ Moreover, septic AKI is independently associated with higher odds of death and longer duration of hospitalization. ${ }^{17} 10$ (9.52\%) patients developed AKI following septicaemia in our study.

Acute pyelonephritis is a potentially organ and/or life threatening infection that characteristically causes some scarring of the kidney with each infection and may lead to significant damage to the kidney, sepsis, or sepsis syndrome/shock/multiorgan system failure. More than 2,50,000 cases occur in the United States each year (1995 estimate), and approximately 200,000 patients require hospitalization (1997 data). ${ }^{18}$ Wide variation exists in the clinical presentation, severity, options, and disposition of acute pyelonephritis. Lower UTIs predispose to pyelonephritis. Diagnosing and managing acute pyelonephritis is not always straightforward. In the age range of 5-65 years, it typically presents in the context of a symptomatic (eg, dysuria, frequency, urgency, gross haematuria, suprapubic pain) urinary tract infection (UTI) with classic upper urinary tract symptoms (eg, flank pain, back pain) with or without systemic symptoms (eg, fever, chills, abdominal pain, nausea, vomiting) and signs (eg, fever, costovertebral angle tenderness) with or without leukocytosis. However, it can present with nonspecific symptoms. ${ }^{19}$ In this study $9(8.57 \%)$ patients developed AKI due to acute pyelonephritis, which is comparable to other studies. ${ }^{18,19}$

Acute kidney injury (AKI) is one of the most dreaded complications of severe malaria. As per World Health Organization criteria, acute kidney injury occurs as a complication of Plasmodium falciparum malaria in less than $1 \%$ of cases, but the mortality rate in these cases may be up to $45 \% .{ }^{20}$ We had 7 (6.66\%) cases of AKI following severe falciparum malaria. Soldiers from operational area in Chittagong hill tracts or African countries were mostly affected. Most of them were admitted during leave.

Contrast-induced nephropathy has become a significant source of hospital morbidity and mortality with the ever-increasing use of iodinated contrast media in diagnostic, imaging and interventional procedures such as angiography in high-risk patients. It is the third most common cause of hospitalacquired acute renal failure, after surgery and hypotension in western countries ${ }^{21}$ which is a sharp contrast to our study.

Incidence of post operative AKI varies from $1.1-17 \%^{22}$. It remains a leading cause of morbidity, mortality prolonged hospital stay and increased hospital cost ${ }^{23}$. We had $5(4.76 \%)$ cases of postoperative AKI, who had normal renal function preoperatively.

In this study $66.66 \%$ patients presented with oliguria and $10.47 \%$ patients presented with anuria. Only 24 $(22.85 \%)$ patient in this study had non-oliguric renal failure.

Twenty five (23.8\%) patients had dehydration at presentation indicates that proper replacement of fluid loss could not made in many cases.

History and clinical examinations are sufficient for making an etiological diagnosis in most of the cases. Among laboratory investigations, blood biochemistry is the main tool for diagnosing AKI.

On routine blood examination in our study $\mathrm{Hb} \%$ was $11.23 \pm 2.9 \mathrm{gm} / \mathrm{dl}$ was not of any diagnostic help and was only useful in planning management especially in case of blood loss. Total and differential leucocyte count revealed polymorphonuclear leucocytosis in 58 $(55.2 \%)$ cases indicating infection. 
Proteinuria, which was the most common indicator of renal pathology found in 44 (41.90\%) cases. Haematuria found in 34 (32.38\%) cases which was due to glomerulonephritis or UTI. Pyuria found in 42 $(40 \%)$ cases indicative of underlying urinary tract infection, many of which developed in the hospital during treatment, RBC cast found in 28 (26.66\%) cases gave important clue to glomerulonephritis(tableIV).

Blood urea, serum creatinine and serum electrolytes are the most essential guide for diagnosis and management of AKI patients. Maximum mean urea level ( $91 \pm 34 \mathrm{mg} / \mathrm{d} 1)$ and creatinine level (4.29 \pm 2.55 $\mathrm{mg} / \mathrm{d} 1$ ) observed in this study(table-V) is comparable to that of Razzak et al study which was $61.07 \pm 23.26$ $\mathrm{mg} / \mathrm{d} 1$ and $14.7 \pm 7 \mathrm{mg} / \mathrm{dl}$ respectively. ${ }^{8}$ Electrolyte imbalance was almost invariable in all patients.

Haemodialysis was done in $42.85 \%$ patients which was the sheet anchor of patient management in this study(table-VII). Furosemide along with conservative management was given to $43.80 \%$ patients with positive results. Continuous renal replacement therapy (CVVHD) was given to 6 haemodynamically unstable patient, 3 of them died due to multiorgan failure and other three recovered successfully. Only 5 patients received peritoneal dialysis due to hypotension or other contraindication to haemodialysis.

Infections were commonest complication of AKI in this study. $67.2 \%$ patients had to be treated with antibiotics. Since infection still remains the primary cause of death, it is possible that the uraemic state predispose to these infections.

Result of treatment in this study was rewarding. 88 (83.80\%) patients were fully recovered. Only $3(2.85 \%)$ died. $8(7.61 \%)$ patients developed some impairment of renal function and $7(6.66 \%)$ developed ESRD. The mortality rate was $2.85 \%$ which is in contradiction to some western literature where mortality is $28-50 \% .^{24}$

The reason for the rewarding result in this study may be due to large number of pre-renal etiology correctable by simple conservative measures, early reporting, immediate and better management .

\section{Conclusion}

Acute kidney injury is potentially life-threatening and requires intensive treatment. Over the year, the etiology and outcome of AKI is changing from country to country and centre to centre. However, the kidneys usually start working again within several weeks to months after the underlying cause has been treated. ${ }^{26}$ In some cases, chronic renal failure or end- stage renal disease may develop. Death is most common when kidney failure is caused by surgery, trauma, or severe infection in someone with heart disease, lung disease, or recent stroke. ${ }^{26,27}$ Old age, infection, loss of blood from the intestinal tract, and progression of kidney failure also increase the risk of death. ${ }^{28}$ Unlike western countries, our cases were mostly pre-renal and preventable. Acute gastroenteritis was the commonest cause of AKI in this study and then next common was NSAIDs. Perhaps appropriate intervention in earlier stages of AKI may have a positive impact on patient outcome and can reverse the process in most cases and therefore the mortality can be in the acceptable range. 29

\section{Conflicts of interest: None}

\section{References:}

1. Webb S, Dobb G "ARF, ATN or AKI? It's now acute kidney injury". Anaesthesia and Intensive Care. (December 2007). 35 (6): 843-4.

2. Brenner and Rector's The Kidney. Philadelphia: Saunders. 2007. ISBN 1-4160-3110-3.

3. Mehta RL, Kellum JA, Shah SV, et al. "Acute Kidney Injury Network: report of an initiative to improve outcomes in acute kidney injury". Critical Care (London, England). (2007). 11 (2): R31.

4. Bellomo R, Ronco C, Kellum JA, Mehta RL, Palevsky $\mathrm{P}$ "Acute renal failure - definition, outcome measures, animal models, fluid therapy and information technology needs: the Second International Consensus Conference of the Acute Dialysis Quality Initiative (ADQI) Group". Crit Care. (2004). 8 (4): R204-12.

5. Palevsky PM, Zhang JH, O'Connor TZ, et al. "Intensity of renal support in critically ill patients with acute kidney injury". The New England Journal of Medicine. (July 2008). 359 (1): 7-20.

6. Schrier RW, Wang W, Poole B, Mitra A "Acute renal failure: definitions, diagnosis, pathogenesis, and therapy". J. Clin. Invest. (2004). 114 (1): 5-14.

7. Prakash J, Tripathi K, Malhotra V, Kumar O, Srivastava PK. Acute renal failure in eastern India. Nephrol Dial Transplant. 1995 Nov;10(11):2009-12.

8. Razzak A,Ahmed S,Rahman M, Profile of acute renal failure in adults. Bang Renal Journal 1983;2:5-8.

9. Nurul M, Harun R, Matiur R. Clinical spectrum of acute renal failure. IPGMR, Dhaka 1987.

10. Rashid HU,Akhter F,Ahmed E.Outcome of acute renal failure in Bangladesh.Renal Failure 1993 ; 15(5):603.

11. Perez S, Garcia LA, Raiford DS, et al.Non steroidal anti inflammatory drugs and risk of hospitalization for ARF. Arch Intern Med.1996;156,2433-243 
12. Huerta C, Castellsague J, Varas-Lorenzo C, García Rodríguez LA .Nonsteroidal anti-inflammatory drugs and risk of ARF in the general population.Am $\mathrm{J}$ Kidney Dis. 2005;45(3):531.

13. Senert R, Kohl L, Rainone T, Scalea T: Exerciseinduced rhabdomyolysis and ARF. Ann Emerg Med June 1994;23:1301-6.

14. Ron D, Taitelman U, Michaelson M, et al: Prevention of acute renal failure in traumatic rhabdomyolysis. Arch Intern Med 1984;144:277-280.

15. Bywaters EG, Beall D "Crush injuries with impairment of renal function" Br Med J (1941). 1 (1): 427-32. doi:10.1136/bmj.1.4185.427.

16. Yegenaga I, Hoste E, Van Biesen W, et al. Clinical characteristics of patients developing ARF due to sepsis/systemic inflammatory response syndrome: resultsof a prospective study.Am J Kidney Dis 2004, 43:817-824.

17. Oppert M, Engel C, Brunkhorst FM,et al: Acute renal failure in patients with severe sepsis and septic shock a significant independent risk factor for mortality: results from the German Prevalence Study. Nephrol Dial Transplant 2008, 23:904-909.

18. Czaja CA, Scholes D, Hooton TM, Stamm WE "Population-based epidemiologic analysis of acute pyelonephritis”. Clin. Infect. Dis(2007).. 45 (3): 27380 .

19. Ramakrishnan K, Scheid DC "Diagnosis and management of acute pyelonephritis in adults". Am Fam Physician. (2005). 71 (5): 933-42.

20. Saroj K. Mishra, MD, Bhabani S, Das B S, Malaria and Acute Kidney Injury . Semin Nephrol. , 2008.28:395-408
21. Tadhg G. Gleeson; Sudi Bulugahapitiya, ContrastInduced Nephropathy, Am J Roentgenol. 2004;183(6) , 2004

22. Kheterpal S, Tremper KK, Englesbe MJ, et al. Predictors of post-operative renal failure after non cardiac surgery in patient with previously normal renal function. Anaesthesiology 2007,107: 892902.

23. Reddy VG: Prevention of post-operative acute renal failure .J postgraduate med 2002,48: 64-70.

24. Woodrow G, Turney JH. Cause of death in acute renal failure. Nephrol Dial Transplant 1992;7:230234.

25. Hoque M, Chowdhury D, Khan MR. Outcome of acute renal failure in children. Bang $J$ of child health 1985; 9(1) 5-10.

26. Papadakis, Maxine A.; McPhee, Stephen J. (2008).Current Medical Diagnosis and treatment. McGraw-Hill Professional..

27. Pannu N, Klarenbach S, Wiebe N, Manns B, Tonelli M (February 2008). "Renal replacement therapy in patients with acute renal failure: a systematic review". JAMA : the J Am Med 299 (7): 793-805.

28. Bellomo R, Cass A, Cole L, et al. (October 2009). "Intensity of continuous renal replacement therapy in critically ill patients". New Eng J Med 361 (17): 1627-3.

29. Cole L, Bellomo R, Silvester W, et al. A prospective, multicentre study of the epidemiology, management and outcome of severe acute renal failure in "closed" ICU system. Am J Respir Crit Care Med 2000; 162: 191-196. 